\title{
Erratum: Higgs properties in a softly broken Inert Doublet Model
}

Rikard Enberg, ${ }^{a}$ Johan Rathsman ${ }^{b}$ and Glenn Wouda ${ }^{a}$

${ }^{a}$ Department of Physics and Astronomy, Uppsala University, Box 516, SE-751 20 Uppsala, Sweden

${ }^{b}$ Department of Astronomy and Theoretical Physics, Lund University, SE-223 62 Lund, Sweden

E-mail: Rikard.Enberg@physics.uu.se, Johan.Rathsman@thep.lu.se, Glenn.Wouda@physics.uu.se

ERRATUM TO: JHEP08(2013)079

ArXiv EPRINT: 1304.1714 
We have discovered that the realization of soft $\mathbb{Z}_{2}$ symmetry breaking in this paper was incorrect, and in fact the symmetry is in our setup also broken by dimension four terms, see our later paper [1] for more details. In brief, the $\mathbb{Z}_{2}$ symmetry is now manifest in the Yukawa sector, but broken in the scalar potential. This does not change the phenomenology of the model, but the motivation is different. The model remains the same, with the exception that equations (2.12) and (2.13) in the published paper are no longer relevant constraints on the parameters, and in our follow-up paper [1] we therefore relax these constraints. The model can be considered as a limit of the aligned two-Higgs doublet model with one fermiophobic doublet [2], and large FCNC are still only generated at the two-loop level.

Therefore, the following changes and corrections should be made to the published paper:

1. All references to "soft" breaking of the symmetry should be ignored. The symmetry is not broken softly in the literal sense of the term, but rather it is manifest in the Yukawa Lagrangian and broken in the scalar potential. This leads to the same phenomenology up to two-loop level.

2. All constraints on the parameters from the incorrect requirement of soft $\mathbb{Z}_{2}$ breaking should be ignored. Specifically, equations (2.12) and (2.13) should be removed. As a consequence, $\lambda_{7}^{\max }$ is no longer relevant in the parameter scans. We have found that the range $-5 \leq \lambda_{7} \leq \min \left\{5, \lambda_{7}^{\max }\right\}$ used in the scan shown in figure 3 makes a small difference compared to using $-5 \leq \lambda_{7} \leq 5$.

3. In eq. (2.14), $\lambda_{2}$ should be added as a parameter. Note that $\lambda_{2}$ does not enter any relevant couplings or masses and only affects the phenomenology indirectly by limiting the values of the other parameters through its effect on theoretical constraints.

Note that in this paper we chose $\lambda_{1}=\lambda_{2}$ as a way to fulfill eq. (2.12). All results obtained from the model in this paper are still valid under this reasonable simplification. In [1] we obtain more detailed results from the model. In particular, figures 4 and $5 \mathrm{a}$ in [1] show the corresponding result as figure 3 in the present paper but taking the newest LHC data into account.

Open Access. This article is distributed under the terms of the Creative Commons Attribution License (CC-BY 4.0), which permits any use, distribution and reproduction in any medium, provided the original author(s) and source are credited.

\section{References}

[1] R. Enberg, J. Rathsman and G. Wouda, Higgs phenomenology in the Stealth Doublet Model, arXiv: 1311.4367 [INSPIRE].

[2] V. Ilisie and A. Pich, Low-mass fermiophobic charged Higgs phenomenology in two-Higgs-doublet models, JHEP 09 (2014) 089 [arXiv:1405.6639] [INSPIRE]. 